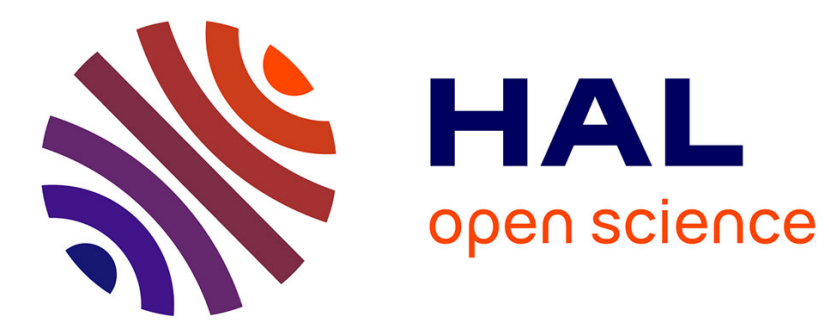

\title{
Time Reversal Receive Antenna Shift Keying On MIMO LOS Channel
}

\author{
Ali Mokh, Yvan Kokar, Maryline Hélard, Matthieu Crussière
}

\section{To cite this version:}

Ali Mokh, Yvan Kokar, Maryline Hélard, Matthieu Crussière. Time Reversal Receive Antenna Shift Keying On MIMO LOS Channel. International Conference on Sensors, Networks, Smart and Emerging Technologies, Sep 2017, Beirut, Lebanon. hal-01562221

\section{HAL Id: hal-01562221 \\ https://hal.science/hal-01562221}

Submitted on 13 Jul 2017

HAL is a multi-disciplinary open access archive for the deposit and dissemination of scientific research documents, whether they are published or not. The documents may come from teaching and research institutions in France or abroad, or from public or private research centers.
L'archive ouverte pluridisciplinaire HAL, est destinée au dépôt et à la diffusion de documents scientifiques de niveau recherche, publiés ou non, émanant des établissements d'enseignement et de recherche français ou étrangers, des laboratoires publics ou privés.

$$
\text { Copyright }
$$




\title{
Time Reversal Receive Antenna Shift Keying On MIMO LOS Channel
}

\author{
Ali Mokh, Yvan Kokar, Maryline Hélard, Matthieu Crussière \\ IETR, INSA de Rennes, France
}

\begin{abstract}
Receive Antenna Shift Keying (RASK) is a Spatial Modulation (SM) transmission scheme that has recently been under study because of its low detection complexity and its ability to increase the spectral efficiency when combined with classical IQ modulation schemes. The idea is that the transmitter uses a focusing technique to target one of the receive antennas. The transmitted information is coded depending on the index of the receive antenna that have been targeted. Note that in this case, the sent signal does not contain any information, that's why RASK can be combined with any classical IQ modulation. Different focusing techniques could be used to target a receive antenna. In this study, the Time Reversal preprocessing technique is used at the transmitter side. In this paper, theoretical performance of the Bit Error Rate and of the focusing gain are established for the RASK scheme in a MIMO Line-of-Sight configuration with two receive antennas. Also, a testbed is evaluated and used to provide RASK performance in realistic conditions.

Index Terms-Receive Antenna Shift Keying, MIMO, Spatial Modulation, Line of Sight, Time Reversal, Pre-coding, Focusing, Preprocessing
\end{abstract}

\section{INTRODUCTION}

Spatial Modulation (SM) has recently established itself as a promising transmission concept consisting in exploiting the index of transmit antennas to transmit additional information bits. In such techniques, location-dependent spatial information is utilized to carry additional information bits thereby boosting the overall spectral efficiency (SE) and overcoming inter-channel interference (ICI). MIMO systems employing SM, which are the so-called SM-MIMO systems, takes advantage of the whole antenna array at the transmitter, while using a limited number of RF chains [1]. The main distinguishing feature of SM-MIMOs is that they map additional information bits onto an "SM constellation diagram", where each constellation element is constituted by either one or a subset of antenna elements [2].

One popular SM scheme at the transmitter side is Space Shift Keying (SSK) [3] whose main principle is to take advantage of the various propagation characteristics associated to the different antennas of the system [4]. Given a rich scattering environment, the receiver can utilize the distinct received signals from different antennas to discriminate between the transmitted information messages. In SM-MIMO systems, the single-stream transmission scheme is combined with the SSK scheme, which facilitates a simplified transmitter with a single $\mathrm{RF}$ front end. The signal processing at the receiver can also be simplified as data are transmitted through a single spatial stream in SM-MIMO systems [5].
Furthermore, a particular SM implementation case proposed in [6] and referred to as Transmitter Preprocessing Aided Spatial Modulation (TPSM), where the SM concept is applied at the receiver side, using Zero-Forcing preprocessing and joining conventional amplitude-phase modulation and preprocessing aided SSK (pre-SSK).

Another receiver SM scheme was proposed in [7] and [8] and referred to as receive antenna shift keying (RASK), where the transmitted signal carries no useful information at the level of the IQ mapping stage, and Time Reversal (TR) preprocessing is used to concentrate the signal energy towards a targeted antenna. TR enables to focus a signal in time and space, and requires a channel state information at the transmitter, and was considered suitable for Time Division Duplex (TDD) systems [9].

With RASK, a simple demodulation can be carried out based on the estimation of the index of RA that receives the maximum real part for a coherent detection, or the maximum power for incoherent detection. Since one single antenna is targeted at a time, the spectral efficiency is however limited to $\log _{2} N_{r}$, with $N_{r}$ the number of receive antennas (RAs).

In this paper, the RASK scheme with TR preprocessing technique in a MIMO Line of Sight (LOS) channel is considered, since in millimeter waves, the channel is considered as mainly LOS. The incoherent detector that compares the amount of received power on each RA is used at the receiver side, because of its simplicity, and because it doesn't need any phase synchronization. Analytical performance of the Bit Error Rate (BER) was derivated, a testbed was implemented to measure the performance in specific conditions of LOS channel, and numerical graphs are shown to evaluate the performance of the system.

The rest of the paper is organized as follows. In Section II, the system model and the block diagram of the RASK scheme are detailed. This is followed, in Section III, by analytic study of the BER performance using incoherent detection. Testbed and numerical results are provided in Section IV, and a conclusion is drawn in Section V.

\section{SYSTEM MODEL FOR RASK}

\section{A. System Model}

A MIMO system with $N_{t}$ transmit antennas (TAs) and $N_{r}$ RAs is considered. Assuming a deterministic MIMO LOS channel taking into account the free space propagation effect on the signal, $\mathbf{H}=\left(h_{j, i}\right)_{1 \leq i \leq N_{t}, 1 \leq j \leq N_{r}}$ where $\mathbf{H} \in \mathbb{C}^{N_{r} \times N_{t}}$ and $h_{j, i}$ represents the complex channel coefficient between 


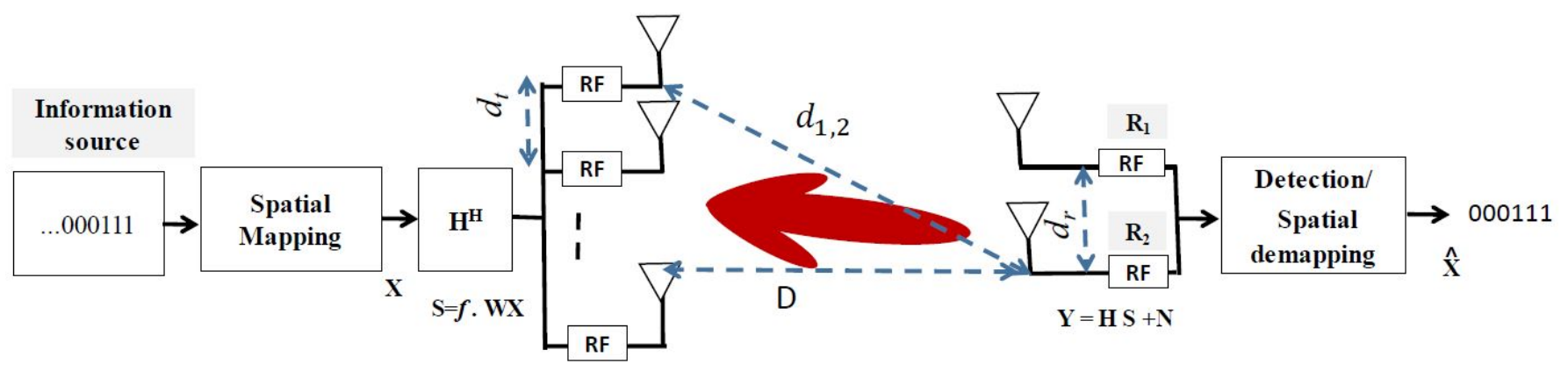

H

Figure 1. Block diagram of RASK

the $i$-th TA, denoted by $T x_{i}$, and the $j$-th RA, denoted by $R x_{j}$. Let:

$$
\tilde{h}_{j, i}=\left(\frac{\lambda}{2 \pi d_{i, j}}\right)^{2} e^{j \theta}
$$

with $\theta=2 \pi d_{i, j} / \lambda, \lambda$ is the wavelength of the wave used, and $d_{i, j}$ the euclidean distance between $T x_{i}$ and $R x_{j}$, be the complex non normalized channel coefficient. Let also:

$$
\beta=\left(\frac{\lambda}{2 \pi D}\right)^{2} e^{j \theta_{0}}
$$

where $\theta_{0}=2 \pi D / \lambda$, and $D$ the distance between the transmitter and the receiver, be the normalization factor. The normalized MIMO-LOS coefficients are then given by $h_{j, i}=\tilde{h}_{j, i} / \beta$. The received signal vector can be written as

$$
\mathbf{Y}=\mathbf{H} . \mathbf{S}+\mathbf{N}
$$

$\mathbf{Y} \in \mathbb{C}^{N_{r} \times 1}$ is the vector of the received signals on all RAs, $\mathbf{S} \in \mathbb{C}^{N_{t} \times 1}$ is the vector of the transmitted signals by all emitting antennas, and $\mathbf{N} \in \mathbb{C}^{N_{r} \times 1}$ is the vector of additive white Gaussian noise (AWGN) samples $\eta_{j}$ at the such that $\eta_{j} \sim \mathcal{N}\left(0, \sigma_{n}^{2}\right)$. An incoherent detection is used in this system, so the additive noise is assumed to be real, as well as the received signal.

\section{B. Transmission Block Diagram}

The block diagram of the RASK system is depicted in Figure 1. A group of $m=\log _{2}\left(N_{r}\right)$ bits is mapped to a spatial symbol $\mathbf{X}_{k} \in \mathbb{R}^{N_{r} \times 1}$ which is written as:

$$
\mathbf{X}_{k}=\left[\begin{array}{lllll}
0 & \ldots & \underbrace{x}_{k_{\text {th }} \text { position }} & \ldots & 0
\end{array}\right]^{T}
$$

where $\mathrm{k}$ is the position of the targeted antenna. Then, the preprocessing block transforms the vector of spatial symbols $\mathbf{X}$ into a vector of transmitted signals denoted as $\mathbf{S} \in \mathbb{C}^{N_{t} \times 1}$ using the preprocessing matrix $\mathbf{W} \in \mathbb{C}^{N_{t} \times N_{r}}$. In this paper, the Time-Reversal (TR) technique is employed, where the transconjugate of the channel matrix is used as a pre-filter:

$$
\mathbf{W}=\mathbf{H}^{H}
$$

Consequently, the transmitted signal is written as:

$$
\mathbf{S}=f \cdot \mathbf{W} \cdot \mathbf{X}
$$

where $f=1 / \sqrt{\sigma_{x}^{2} \operatorname{Tr}\left(\mathbf{H}^{H} \cdot \mathbf{H}\right)}$ is a normalization factor used to guarantee that the average total transmit power $\bar{P}_{t}$ is equal to $1, \operatorname{Tr}($.$) holds for the trace of matrix and \mathbb{E}_{x}$ stands for the expectation over $x$. Since $\mathbf{X}$ has i.i.d. entries, the variance $\sigma_{x}^{2}=\mathbb{E}_{x}\left[x_{j} x_{j}^{*}\right]$ is independent of $j$ and comes in factor of the trace computation. Then, only one of $N_{r}$ entry of $\mathbf{X}$ is of amplitude $\sqrt{E_{S}}$ with a probability that can be easily verified to be of $\frac{1}{N_{r}}$, leading to $\sigma_{x}^{2}=\frac{E_{S}}{N_{r}}$.

\section{Received Signal}

At the level of the RA $R x_{j}$, the received signal then simply writes:

$$
y_{j}=f \times \sum_{i=1}^{N_{t}} h_{j, i} \cdot h_{k, i}^{*} \times x+\eta_{j}
$$

and for $j=k$, we have:

$$
\begin{aligned}
y_{k} & =f \times \sum_{i=1}^{N_{t}} h_{k, i}^{2} \times x+\eta_{k} \\
& \approx f \times N_{t} x+\eta_{k}
\end{aligned}
$$

Or, for the non targeted antennas, the received amplitude is the interference amplitude, which can be given after mathematical derivation by:

$$
y_{j, j \neq k} \approx f\left(\frac{\sin \left(\pi N_{t} G\right)}{\sin (\pi G)}\right) x+\eta_{j} \text { where } G=\frac{d_{t} d_{r}}{D \lambda}
$$

Let $A_{S}=f \cdot N_{t}$ and $A_{I}=f\left(\frac{\sin \left(\pi N_{t} G\right)}{\sin (\pi G)}\right)$, and define $S I R$ the signal to interference ratio, which is the ratio of the useful received power on the targeted antenna and the interference received power on the non targeted antenna. We have:

$$
S I R=\left(\frac{A_{S}}{A_{I}}\right)^{2}=\left(\frac{\sin (\pi G) \times N_{t}}{\sin \left(\pi N_{t} G\right)}\right)^{2}
$$




\section{AnAlyticAl STUdy}

In this paper, a non coherent detector is used, where the index of the targeted antenna is estimated as:

$$
\begin{gathered}
\hat{k}=\operatorname{Arg} \max _{j}\left\|y_{j}\right\|^{2} \\
\forall j \in\left[1 ; N_{r}\right],\left\|y_{j}\right\|^{2} \leq\left\|y_{\hat{k}}\right\|^{2}
\end{gathered}
$$

The BER of a two receive antenna RT-RASK scheme using the above receiver is given by:

$$
\mathcal{P}_{e}=\frac{1}{2}\left(\mathcal{P}\left(\mathbf{X}_{1} \rightarrow \mathbf{X}_{2}\right)+\mathcal{P}\left(\mathbf{X}_{2} \rightarrow \mathbf{X}_{1}\right)\right)
$$

where $\mathcal{P}\left(\mathbf{X}_{i} \rightarrow \mathbf{X}_{j}\right)$ is the probability to detect $X_{j}$ such that $X_{i}$ was transmitted. Let $\mathcal{X}_{i, j}=\left|y_{j} / X_{i}\right|$ which is the square root of the measured power at the $j_{t h}$ RA such that the $i_{t h}$ antenna was targeted.

$$
\mathcal{P}_{e}=\frac{1}{2}\left(\mathcal{P}\left(\mathcal{X}_{1,1}<\mathcal{X}_{2,1}\right)+\mathcal{P}\left(\mathcal{X}_{2,2}<\mathcal{X}_{1,2}\right)\right)
$$

Since

$$
\begin{aligned}
& \mathcal{X}_{1,1} \text { and } \mathcal{X}_{2,2} \sim\left|\mathcal{N}\left(A_{S}, \sigma_{n}^{2}\right)\right| \\
& \mathcal{X}_{1,2} \text { and } \mathcal{X}_{2,1} \sim\left|\mathcal{N}\left(A_{I}, \sigma_{n}^{2}\right)\right|
\end{aligned}
$$

we have:

$$
\begin{aligned}
\mathcal{P}_{e} & =\mathcal{P}\left(\left|\mathcal{N}\left(A_{S}, \sigma_{n}^{2}\right)\right|<\left|\mathcal{N}\left(A_{I}, \sigma_{n}^{2}\right)\right|\right) \\
& =\int_{-\infty}^{+\infty} f_{\mathcal{X}_{1,1}}(x) \cdot\left(1-F_{\mathcal{X}_{2,1}}(x)\right) d x
\end{aligned}
$$

where:

$$
f_{\mathcal{X}_{1,1}}(x)=\frac{1}{\sqrt{2 \pi \sigma_{b}^{2}}}\left(e^{\frac{-\left(x-A_{S}\right)^{2}}{2 \sigma_{b}^{2}}}+e^{\frac{-\left(x+A_{S}\right)^{2}}{2 \sigma_{b}^{2}}}\right)
$$

is the probability density function of $\mathcal{X}_{1,1}$, and

$$
F_{\mathcal{X}_{2,1}}(x)=\frac{1}{2}\left[\operatorname{erf}\left(\frac{x+A_{I}}{\sqrt{2} \sigma_{b}}\right)+\operatorname{erf}\left(\frac{x-A_{I}}{\sqrt{2} \sigma_{b}}\right)\right]
$$

is the cumulative density function of $\mathcal{X}_{1,1}$. After some mathematical derivation, the equation 15 becomes:

$$
\mathcal{P}_{e}=\frac{1}{2}\left[\operatorname{erfc}\left(\frac{A_{S}+A_{I}}{\sqrt{2} \sigma_{b}}\right)+\operatorname{erfc}\left(\frac{A_{S}-A_{I}}{\sqrt{2} \sigma_{b}}\right)\right]
$$

\section{NUMERICAL AND EXPERIMENTAL RESUlTS}

\section{A. Numerical results}

The performance of the RT-RASK system on MIMO LOS channel is evaluated through the measurement of the BER versus the ratio between the average transmit power level and noise level, i.e. $\frac{\bar{P}_{t}}{\sigma_{n}^{2}}$ and for different number of TAs. The power for each sub-channel is normalized:

$$
E\left[\left\|H_{j, i}\right\|^{2}\right]=1
$$

Finally, we consider that the channel response is perfectly known at the transmitter. Simulations are run by implementing a sufficient number of iterations for different channel realizations, and taking the mean value of the BER for each value of $\frac{\bar{P}_{t}}{\sigma^{2}}$.

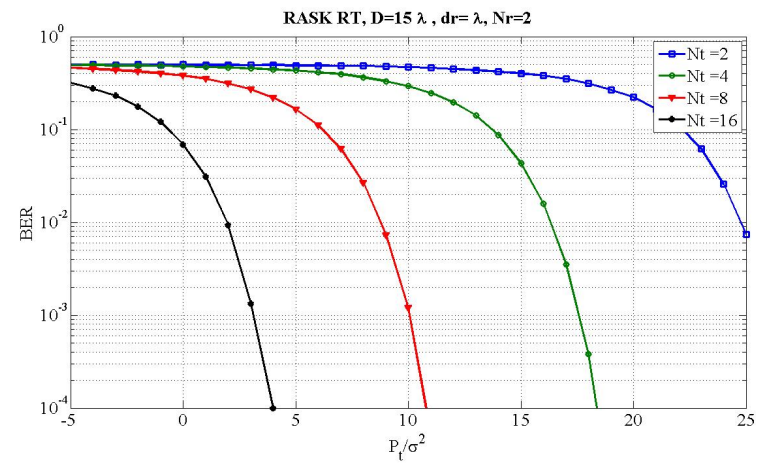

Figure 2. BER numerical result of a MIMO LOS TR-RASK transmission system, for $d_{r}=\lambda, d_{t}=\lambda / 2$ and $D=15 \lambda$

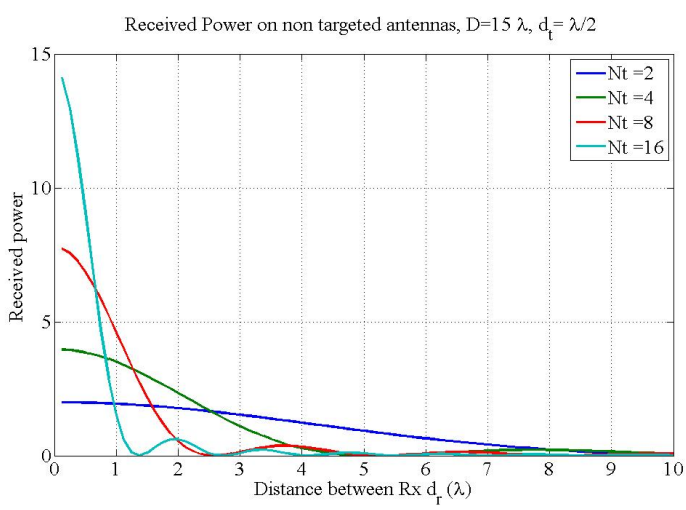

Figure 3. Received power on the non targeted antenna in terms of the distance between RAs

A MIMO RT-RASK with with $N_{r}=2$ and $N_{t}=2,4$, 8 and 16 antennas is considered. The channel is assumed to be LOS, with no fading, and with $D=15 \lambda, d_{t}=\lambda / 2$ and $d_{r}=\lambda$. In Figure 2, the incoherent maximum power detector is used, and the performance based on the analytic study is evaluated. As evident from the obtained curves, increasing the number of the transmit antennas leads to improvement of the performance, because of the increasing in the focusing gain.

In Figure 3, we measured the received power on the non targeted antenna while targeting the other one, for different distance $d_{r}$ between the two antennas. It is shown that the form of the beam change in the distance having local maximum and minimum, and that increasing the number of transmit antennas leads to more directive beam, which explain the improvement of the performance in the Figure 2. Note that because of the normalization of the transmission channel, the amplitude of the interference for $N_{t}=16$ is higher than the others near the targeted antenna, but also the received amplitude at the targeted antenna is also the highest, and the beam is more directive.

\section{B. Experimental Results}

A RASK TDD MIMO transmission system between a transmitter featuring $N_{t}=4$ antennas and a receiver with 


\begin{tabular}{||c|c|c||}
\hline & Theoretical Result & Testbed Result \\
\hline \hline SIR -Targeting RxI & $0.0343[\mathrm{~dB}]$ & $0.0380[\mathrm{~dB}]$ \\
\hline SIR -Targeting $R x 2$ & $0.0343[\mathrm{~dB}]$ & $0.6811[\mathrm{~dB}]$ \\
\hline BER & 0.2028 & 0.2549 \\
\hline
\end{tabular}

TESTBED AND THEORETICAL SIR AND BER RESULTS OF A 4X2 MIMO LOS CHANNEL WITH $\nu=2.4 \mathrm{GHz}, D=15 \lambda, d_{r}=\lambda / 4$ AND $d_{t}=\lambda / 2$.
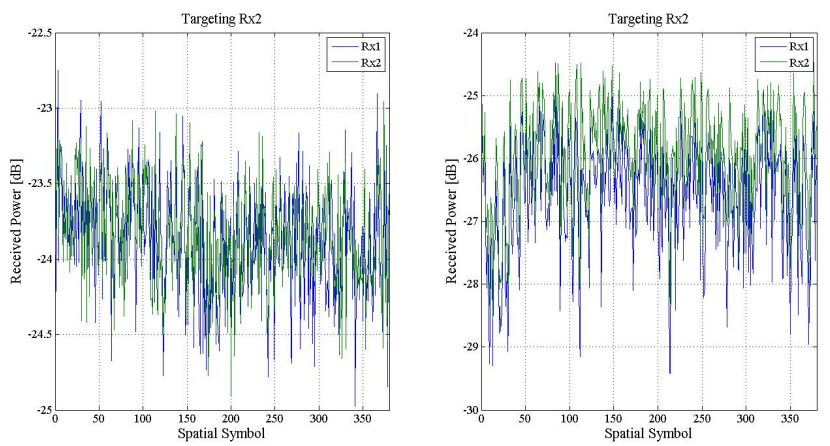

Figure 4. Received power on each antenna targeting Rx1 (Left) and Rx2 (right)

$N_{r}=2$ antennas is considered. The system is implemented using two FPGA-based motherboards (WARP v3), with additional radio board (FMC-RF-2X45) and clock board (CM$\mathrm{MMCX}$ ) for each, configured with the WARPLab Reference Design, as described in [10]. A $125 \mathrm{kbps}$ RASK transmission at $\nu=2.4 \mathrm{GHz}$ is considered in MIMO LOS configuration with $D=15 \lambda, d_{r}=\lambda / 4$ and $d_{t}=\lambda / 2$. A Time Reversal preprocessing technique is used to target the RA. Figure 4 shows the detected power at the two RAs, the blue color for $R x_{1}$ and the green for $R x_{2}$, when targeting $R x_{1}$ (left figure), and $R x_{2}$ (right figure). Table I shows the measurement results and their associated theoretical results for the considered environment. A BER equal to 0.2549 is obtained with the testbed, which is closed to the BER obtained with theoretical approach equal to 0.2028 . Moreover, for the SIR measurement, it is shown that theoretically, a same SIR on the two RAs equal to $0,0343 \mathrm{~dB}$ is resulted, because of the geometry of the system. But with testbed measurement, we calculate the mean of the received power on each antenna during the transmission. $R x_{1}$ gives a result equal to $0.0380 \mathrm{~dB}$ that is close to the theoretical one, while $R x_{2}$ provides a higher SIR equal to $0.6811 \mathrm{~dB}$, and that is caused by some reflections in the environment that will improve the focusing toward this RA.

\section{CONCLUSION}

In this paper, we evaluated the performance of a Receive Antenna Shift Keying using the Time Reversal preprocessing technique at the transmitter, and a simple incoherent power detection at the receiver, over a deterministic MIMO Line of Sight transmission channel, and for two receive antennas. We derive an analytic Bit Error Rate performance of the system, and we calculate the amount of interference on the non targeted antenna. Numerical result have been provided for different number of transmit antennas. We demonstrate that increasing the number of transmit antennas leads to better focusing of the power over targeted antenna, and so to a better performance. Moreover, a real testbed is evaluated and used to measure the performance of the technique on the specified conditions, confirming the theoretical results.

\section{ACKNOWLEDGMENT}

The authors would like to thank the SPATIAL MODULATION project funded by the French National Research Agency (ANR)

\section{REFERENCES}

[1] R. Y. Mesleh, H. Haas, S. Sinanovic, C. W. Ahn, and S. Yun, 'Spatial modulation', IEEE Transactions on Vehicular Technology, vol. 57, no. 4, pp. 2228-2241, 2008.

[2] S. Ganesan, R. Mesleh, H. Ho, C. W. Ahn, and S. Yun, 'On the performance of spatial modulation ofdm', in 2006 Fortieth Asilomar Conference on Signals, Systems and Computers, IEEE, 2006, pp. 1825-1829.

[3] Y. A. Chau and S.-H. Yu, 'Space modulation on wireless fading channels', in Vehicular Technology Conference, 2001. VTC 2001 Fall. IEEE VTS 54th, IEEE, vol. 3, 2001, pp. 1668-1671.

[4] J. Jeganathan, A. Ghrayeb, L. Szczecinski, and A. Ceron, 'Space shift keying modulation for mimo channels', IEEE Transactions on Wireless Communications, vol. 8, no. 7, pp. 3692-3703, 2009.

[5] M. Di Renzo, H. Haas, A. Ghrayeb, S. Sugiura, and L. Hanzo, 'Spatial modulation for generalized mimo: Challenges, opportunities, and implementation', Proceedings of the IEEE, vol. 102, no. 1, pp. 56-103, 2014.

[6] L.-L. Yang, 'Transmitter preprocessing aided spatial modulation for multiple-input multiple-output systems', in Vehicular Technology Conference (VTC Spring), 2011 IEEE 73rd, IEEE, 2011, pp. 1-5.

[7] D.-T. Phan-Huy and M. Hélard, 'Receive antenna shift keying for time reversal wireless communications', in 2012 IEEE International Conference on Communications (ICC), IEEE, 2012, pp. 4852-4856.

[8] A. Mokh, M. Hélard, and M. Crussière, 'Extended receive antenna shift keying', in 2017 IEEE International Conference on Telecommunication (ICT), IEEE, 2017.

[9] A. Derode, P. Roux, and M. Fink, 'Robust acoustic time reversal with high-order multiple scattering', Physical review letters, vol. 75, no. 23, p. 4206, 1995.

[10] Y. Kokar, J.-C. Prevotet, and M. Helard, 'Receive antenna shift keying modulation testbed for wireless communications systems', in Globecom Workshops (GC Wkshps), 2016 IEEE, IEEE, 2016, pp. 1-6. 